\title{
Evaluation Des Risques D’érosion Hydrique Et Cartographie Des Zones Vulnérables Par La Méthode RUSLE Couplée Aux SIG Et À La Télédétection Dans Le Bassin Versant d'Agoudal En Amont De La Vallée d'Imilchil (Haut Atlas Central, Maroc)
}

\section{Youssef Ouadjane (Dr.)}

Equipe Environnement et Valorisation des Ressources Microbiennes et Végétales (EVRMV), Faculté des Sciences Meknès, Université Moulay Ismail, Meknès, Maroc

El-Arbi Ait Yacine (Dr.)

Equipe Environnement et Valorisation des Ressources Microbiennes et Végétales (EVRMV), Faculté des Sciences Meknès, Université Moulay Ismail, Meknès, Maroc

\section{Brahim Benzougagh (Pr.)}

Département de Géomorphologie et Géomatique, Institut Scientifique Université Mohamed V, Rabat, Maroc

\section{Laila Nassiri (Pr.)}

Equipe Environnement et Valorisation des Ressources Microbiennes et Végétales (EVRMV), Faculté des Sciences Meknès, Université Moulay Ismail, Meknès, Maroc

\section{Jamal Ibijbijen (Pr.)}

Equipe Environnement et Valorisation des Ressources Microbiennes et Végétales (EVRMV), Faculté des Sciences Meknès, Université Moulay Ismail, Meknès, Maroc

\section{Doi:10.19044/esj.2021.v17n21p66}

Submitted: 19 April 2021

Accepted: 21 May 2021

Published: 30 June 2021
Copyright 2021 Author(s)

Under Creative Commons BY-NC-ND 4.0 OPEN ACCESS

Cite As:

Ouadjane Y., Ait Yacine E-A., Benzougagh B., Nassiri L. \& Ibijbijen J. (2021). Evaluation Des Risques D’érosion Hydrique Et Cartographie Des Zones Vulnérables Par La Méthode RUSLE Couplée Aux SIG Et À La Télédétection Dans Le Bassin Versant d’Agoudal En Amont De La Vallée d'Imilchil (Haut Atlas Central, Maroc). European Scientific Journal, ESJ, 17(21), 66.

https://doi.org/10.19044/esj.2021.v17n21p66 


\section{Résumé}

Le sous-bassin versant (SBV) d'Agoudal est la partie amont de la vallée d’Imilchil relevant de la province de Midelt et la région de DraaTafilalet au Sud-Est du Maroc. Il s’étale sur la tranche altitudinale asylvatique du Haut Atlas central, allant de 2400 à plus de $3150 \mathrm{~m}$. Ce sont des écosystèmes fragiles dont les sols sont peu arables et peu protégés, à haut risques d'érosion. Ils sont dans un stade très avancé de dégradation, sous l'effet de l'action de l'Homme, des facteurs écologiques, aggravés par les changements climatiques. L'objectif principal de cette recherche est d'évaluer le degré de sensibilité de la zone d'étude vis-à-vis de l'érosion hydrique et de cartographier les zones vulnérables prioritaires pour d'éventuelles interventions d'atténuation. La méthode utilisée s’est basée sur l’Équation Universelle Révisée des Pertes en sols (RUSLE) en intégrant les différents facteurs causaux de ladite équation dans le Systèmes d'Information Géographique (SIG) et en se servant des données officielles (cartes géologiques et topographiques de la zone d'études, données climatiques, les études sur l'érosion réalisées par les départements étatiques concernés) et la télédétection, validées par les réalités de terrain. Les résultats dégagés montrent que la quasi-totalité de ce bassin est soumis à une forte dégradation des sols ; en effet près de $66 \%$ de la superficie de la zone d'étude est couverte par les classes de dégradation spécifique de 50 à 400 t/ha/an et 18.9\% affiche des taux faibles à moyens allant de 7,4 à 32,17 t/ha/an. Seulement 1,4\% du SVB est soumise à des taux de dégradation spécifique inférieurs à 7,4 t/ha/an. La valeur moyenne du taux d'érosion est de 255t/h/an, avec un écart type de près 285 t/an/ha, dû l’hétérogénéité des caractéristiques du milieu et de ses conditions. Ces chiffres attestent que cette région est soumise aux hauts risques d'érosion. Ce phénomène ajouté aux inondations récurrentes, constituent la principale menace qui met en péril l'agriculture vivrière de cette zone, ce qui donne le signal d'alarme pour intervention de mitigation urgente.

Mots clés: Erosion, Perte En Sol, Environnement, Imilchil 


\section{Risk Assessment Of Water Erosion And Mapping Of Vulnerable Areas By The RUSLE Method Coupled With GIS And Remote Sensing In The Agoudal Watershed Upstream Of The Imilchil Valley (High Atlas Central, Morocco)}

\section{Youssef Ouadjane (Dr.)}

Equipe Environnement et Valorisation des Ressources Microbiennes et Végétales (EVRMV), Faculté des Sciences Meknès, Université Moulay Ismail, Meknès, Maroc

\section{El-Arbi Ait Yacine (Dr.)}

Equipe Environnement et Valorisation des Ressources Microbiennes et Végétales (EVRMV), Faculté des Sciences Meknès, Université Moulay Ismail, Meknès, Maroc

Brahim Benzougagh (Pr.)

Département de Géomorphologie et Géomatique, Institut Scientifique

Université Mohamed V, Rabat, Maroc

\section{Laila Nassiri (Pr.)}

Equipe Environnement et Valorisation des Ressources Microbiennes et Végétales (EVRMV), Faculté des Sciences Meknès, Université Moulay Ismail, Meknès, Maroc

\section{Jamal Ibijbijen (Pr.)}

Equipe Environnement et Valorisation des Ressources Microbiennes et Végétales (EVRMV), Faculté des Sciences Meknès, Université Moulay Ismail, Meknès, Maroc

\section{Abstract}

The Agoudal sub-basin is the upstream part of the Assif Melloul watershed in the Imilchil valley belonging to the province of Midelt and the region of Draa-Tafilalet in south-eastern Morocco. It is located on the Asylvatic altitudinal slice of the Central High Atlas, ranging from 2400 to more than $3150 \mathrm{~m}$. These are fragile ecosystems with poor arable land that are poorly protected, with a high risk of erosion. They are in fact in their advanced stage of degradation due to human activities and ecological factors aggravated by climate change. This paper focuses on assessing the degree of sensitivity of this area to water erosion, and it aims to map priority vulnerable areas for any future mitigation intervention. The method was based on the Revised Universal Soil Loss Equation (RUSLE) by integrating the causal factors of this equation in Geographic Information Systems (GIS) and by using remote sensing data validated based on official data (geological maps and topography of the study area, climatic data, studies on erosion carried out by the state departments concerned) and remote sensing (validated by the realities on the 
ground). The results show that almost all the watershed is subject to severe soil degradation due to water erosion. In fact, nearly $66 \%$ of its area is covered by specific degradation classes of 50 to $400 \mathrm{t}$ / ha / year, and $18.9 \%$ of the area displays low and medium erosion rates. Only $1.4 \%$ of the study area is subject to specific degradation rates less than $7.4 \mathrm{t} / \mathrm{ha} /$ year. The average erosion rate is $255 \mathrm{t} / \mathrm{h} /$ year, with a standard deviation of $285 \mathrm{t} /$ year / ha, mainly due to the heterogeneity of the characteristics and its conditions. These figures show that this region is subject to high risks of erosion. This phenomenon, along with recurrent floods, constitutes the main threat that is endangering subsistence agriculture, which gives the alarm signal for urgent mitigation intervention.

Keywords: Erosion, Soil loss, RUSLE, Environment, Imilchil

\section{Introduction}

L'érosion hydrique et la dégradation des sols constituent un processus de dégradation des ressources naturelles nationales, sous l'effet combiné des facteurs naturels et l'action anthropique. À l'instar des autres pays méditerranéens, Le Maroc souffre activement de ce problème qui touche environ 43\% de son territoire (FAO, 1996). La disparition de la couche arable des sols marocains est estimée à environ 22000 ha/an (Croitoru et al., 2017) et que plus de 12000000 d'hectares dont 1/3 des terres cultivables sont menacés de dégradation par érosion (Benbrahim et al., 2004). Ainsi, sur 23 millions d'hectares en zones montagneuses, $75 \%$ sont touchées par l'érosion dont un tiers de manière très critique. (Badraoui et al., 2001; Benzougagh et al., 2020). Les activités humaines (surpâturage, déboisement, urbanisation) et les facteurs naturels liés essentiellement aux changements climatiques dégradent ces zones de multiples manières. En effet sur les terres cultivées, la dégradation affecte environ 5,5 millions d'hectares de zones pluviales (en raison de l'érosion) et 160000 ha de terres irriguées par salinisation ; ces pertes sont estimées à 1,7 milliards de DH (Croitoru et al., 2017). Ce problème affecte également les parcours, à travers le défrichage (65000 ha/an), la désertification (103000 ha /an) et la surexploitation (30,2 millions ha/an) (Croitoru et al., 2017).

$\mathrm{Au}$ haut Atlas Marocain, la situation continue à se détériorer et engendredes taux de dégradation spécifique des sols allant de 500 à 1000 t $/ \mathrm{km}^{2} / \mathrm{an}$ (Ghanam, 2003) et affecte la qualité des sols en induisant la détérioration de leurs couches superficielles riches en matière organique conduisant à la perte de productivité et fertilité des sols et à la dégradation des terres (Modeste et al., 2016). Ces chiffres déclenchent l'alarme aux responsables et décideurs pour intervention urgente, vu les coûts environnementaux et socio-économiques de plus en plus élevés. 
Dans le cadre du présent travail nous proposons une méthodologie de cartographie des zones vulnérables au risque d'érosion hydrique en amont du bassin versant (BV) d'Oum Er-Rabia, plus exactement au niveau du sous bassin (SBV) d'Agoudal, un affluant de l'Oued d'Assif Melloul dans le HautAtlas central marocain. C'est une zone asylvatique où l'érosion et les inondations affectent aussi bien le milieu naturel que le milieu humain et présentent de sérieux problèmes dans le fonctionnement des écosystèmes de la vallée d'Imilchil en induisant la réduction de la superficie des sols agricoles, déjà peu arables, et en accélérant l'envasement des infrastructures hydrauliques et routières en aval. Ce qui remet en cause les modes de gestion conventionnelle des bassins versants basé essentiellement sur des aménagements mécaniques transitoires et incite les différents intervenants à investiguer dans la recherche d'alternatives innovantes efficaces et durables pour lutter contre ce phénomène et $\mathrm{y}$ assurer la conservation des sols et la restauration des équilibres écosystémiques. C'est dans cette optique que s'encarte l'objectif de la présente recherche.

Pour réaliser ce travail on s'est basé sur l'intégration des systèmes d'information géographique (SIG) couplés à la télédétection spatiale, en appliquant le modèle empirique RUSLE (Revised Universal Soil Loss Equation), (Renard et al., 1991) sur la base de données de terrain et topographiques. Cela nous a permis de superposer et d'analyser les facteurs synthétisés. Les différents outputs réalisés ont été ensuite confrontés aux données et à la réalité de terrain pour validation.

Le but ultime visé dans le cadre de ce travail est d'identifier les zones vulnérables à haut risques et de cartographier les facteurs d'érosion et de dégradation des sols. Ce qui pourrait contribuer à la constitution d'un outil d'aide à la prise de décision et d'intervention en matière d'aménagement raisonné de cette vallée de montagne, ce qui ne manque pas d'intérêt dans son développement durable.

\section{Matériels et méthodes}

\section{Zone d'étude}

Le Sous Bassin Versant(SBV) d'Agoudal dont la tranche altitudinale allant de 2400 à plus de 3150 se situe à environ $20 \mathrm{~km}$ au sud-est du centre d'Imilchil, en amont du bassin versant d'Assif Melloul dont les eaux courantes déversent dans l'Oued Ahnsal et constituent un réseau hydrographique du Haut Atlas central marocain qui débouche dans la retenue de Bin El Widane (Figure 1). Il couvre une superficie d'environ 6240 ha, cadrée par deux quadrilatères de coordonnées : $(\mathrm{X} 1: 471638, \mathrm{Y} 1: 173013)$ et $(\mathrm{X} 2: 479859$, Y2 : 180436). Il relève administrativement de la collectivité territoriale de Bouzmou, cercle d’Imilchil, province de Midelt dans la région de DraaTafilalet. 
Ce SBV est Caractérisé par ses terrains très accidentés, ses conditions climatiques très rudes et par la dégradation de son couvert végétal, sous l'effet des changements climatiques et l'action zooanthropique, et fournit un cas typique pour le phénomène de l'érosion hydrique.

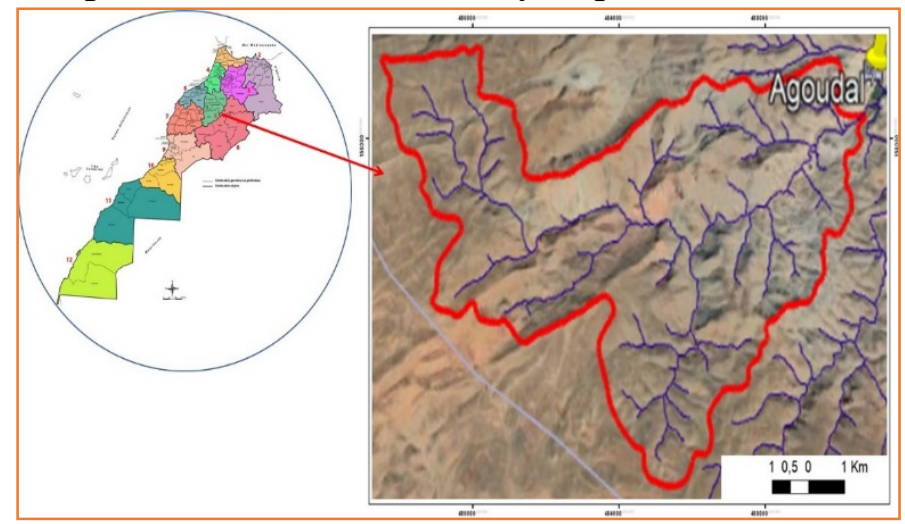

Figure 1. Carte de situation géographique du SBV d'Agoudal

De point de vue géologique (Figure 2), le SBV d'Agoudal est entouré dans ses parties nord et est de grands synclinaux en alternance latérale avec des structures anticlinales étroites(crêtes), avec des amplitudes allant à des centaines de mètres (Michard et al., 2014; Ibouh et al., 2017). Il est situé entre les crêtes d'Ikerzi et de Toumliline. Ces dernières sont principalement orientées N60 vers Est-Ouest et sont carottées par des basaltes du Trias, du gabbro alcalin du jurassique et du calcaire liassique. Ces formations sont largement dominées par les couches marno-calcaires du jurassique (Figure 2) qui s'étendent sur la quasi-totalité de la superficie du BV Assif Melloul comme c'est le cas de toute la chaine du Haut Atlas (Laville et al., 1977). Elles exposent une série sédimentaire de calcaire marin du Liassique au BajocienBathonien, avec des lits rouges répandus dans les strates bathoniennes et les basaltes tholéiitiques (Choubert et al., 1960 ; Jenny et al.,1981). La deuxième série du Jurassique, est composé de dépôt de carbonates et de schistes coiffés de lits rouges terrigènes (Charrière et al., 2016). La série imperméable de Toarcien-Aalénien où s’installent les vallées, se partage la superficie avec la série de calcaires, calcaires- marneux et marnes du Toarcien au Bajocien. Le Jurassique se termine par une série surtout gréseuse, continentale, de faciès rouge (Jurassique supérieur), passant au Crétacé inférieur. Seules les restes des dépôts subaériens d'aleocène supérieur - éocène sont préservés dans des crêtes brisées en raison des épisodes successifs d'érosion (Charrière et al., 2009 ; Michard et al., 2011). Les strates du Crétacé sont composées de calcaire Cénomanien-Turonien et les plus hautes, aussi du Crétacé sont formées de lits rouges terrigènes (Froitzheim et al., 1988; Amrhar, 1995). 
De manière générale, les formations géologiques d'Assif Melloul ont des caractéristiques lithologiques compactes, dures et encroûtées. Celles exposées aux fortes altérations, ont déjà atteint des stades avancés à cause des conditions climatiques agressives associées aux effets amplificateurs des changements climatiques et de l'action anthropique qui ont conduit à la dégradation des terres et aux pertes en sols par l'érosion hydrique (Boudhar et al., 2007).

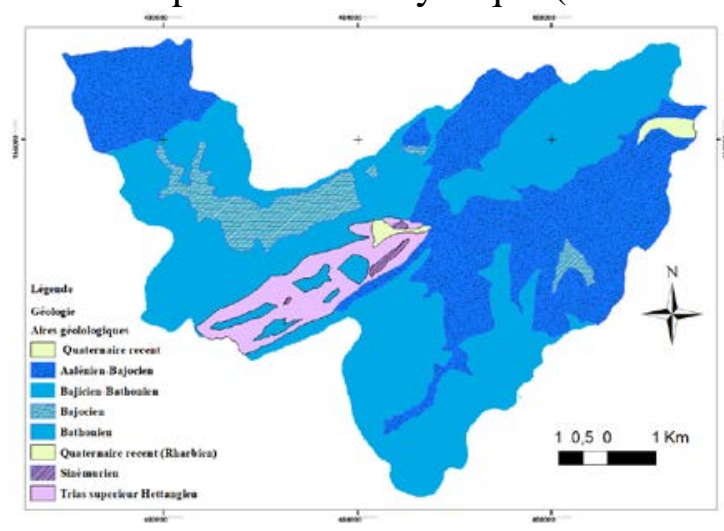

Figure 2. Carte géologique du SBV d'Agoudal

Le climat régnant dans le SBV est caractérisé par un bioclimat semiaride à hiver froid à très froid, avec des chutes de neige et une pluviométrie moyenne de $317 \mathrm{~mm}$ au niveau d'Imilchil. Celle-ci est caractérisée par une variabilité interannuelle importante oscillant entre les années sèches et les années humides. Elles fluctuent de $49.5 \mathrm{~mm}$ /an durant la compagne de 19821983 à 661 mm/an durant la compagne 1995-1996. La température minimale absolue du mois le plus froid atteind $-18^{\circ} \mathrm{C}$ avec une moyenne des minima de $-5.1^{\circ} \mathrm{C}$ et la moyenne des maximas de 32.4C (Mrabet et al. ,2020).

Sur le plan lithologique (Figure 3) la zone d'étude présente une structure fine avec une granulométrie caractérisée par une concentration des limons et des sables fins érodés des versants de charriage.

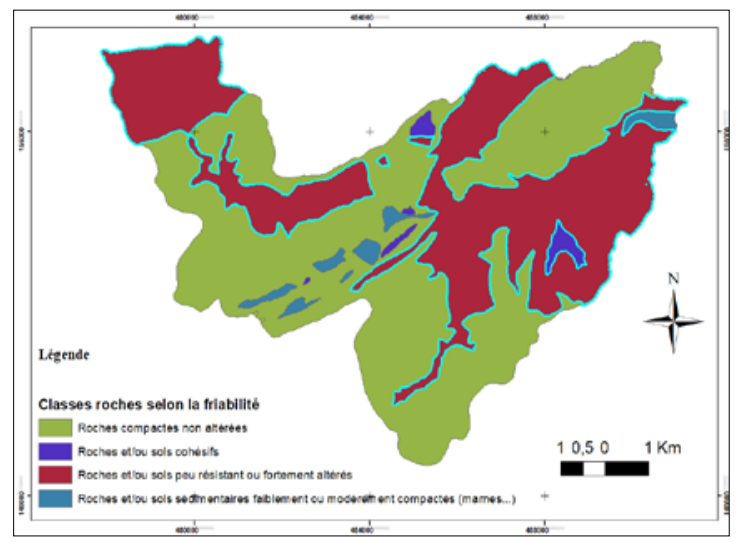

Figure 3. Carte des classes de friabilité des roches de la zone d'étude. 
La quasi-absence de végétation pérenne arbustive ou arborée, fait que les résidus qui restent au sol ne permettent pas de restituer une matière organique pouvant favoriser cette structure des sols, exception faite au niveau des parcelles cultivées. Le Déboisement intensif lié aux activités de pâturage et à la surexploitation du bois par la population (cuisson, chauffage), conjugué aux aléas climatiques aggravent davantage les phénomènes d'érosion et de perte massive en sol.

\section{Méthodologie}

Pour atteindre notre objectif de cartographier la sensibilité des sols à l'érosion hydrique dans le SBV d'Agoudal, nous avons adopté une méthodologie basée sur l'équation universelle révisée de la perte de sol (RUSLE), développée par Renard et al. (1991). Cette approche a été utilisée par plusieurs chercheurs à travers le monde, notamment au Maroc pour évaluer et cartographier le risque d'érosion hydrique (Anys et al., 1992, 1994; Benmoussa et al., 1993; Tahri et al., 1993; El Garouani et al., 2003, 2005, 2008; Merkhi et al., 2015; Elalaoui et al., 2015; Issa et al., 2016; Ouallali et al., 2016 ; Benzougagh et al., 2020). L'équation RUSLE est un modèle qui intègre cinq paramètres de base qui interviennent dans la détermination de ce phénome (Figure 4); il est exprimé dans l'équation (Eq.1) comme suit:

$$
\mathbf{A}=\mathbf{R} * \mathbf{K} * \mathbf{C} * \mathbf{S L} * \mathbf{P}
$$

( Eq.1)

\section{Avec:}

- $\quad \mathbf{A}=$ taux de perte en sol (t/ha/an);

- $\quad \mathbf{R}=$ érosivité des pluies (MJ.mm/ ha.h.an);

- $\quad \mathbf{K}=$ érodibilité du sol (t.h/ha. MJ.mm) ;

- $\quad \mathbf{L S}=$ facteur topographique (L en $\mathrm{m}, \mathrm{S}$ en \%) ;

- $\quad \mathbf{C}=$ facteur de couverture végétale ;

- $\quad \mathbf{P}=$ facteur des pratiques culturales

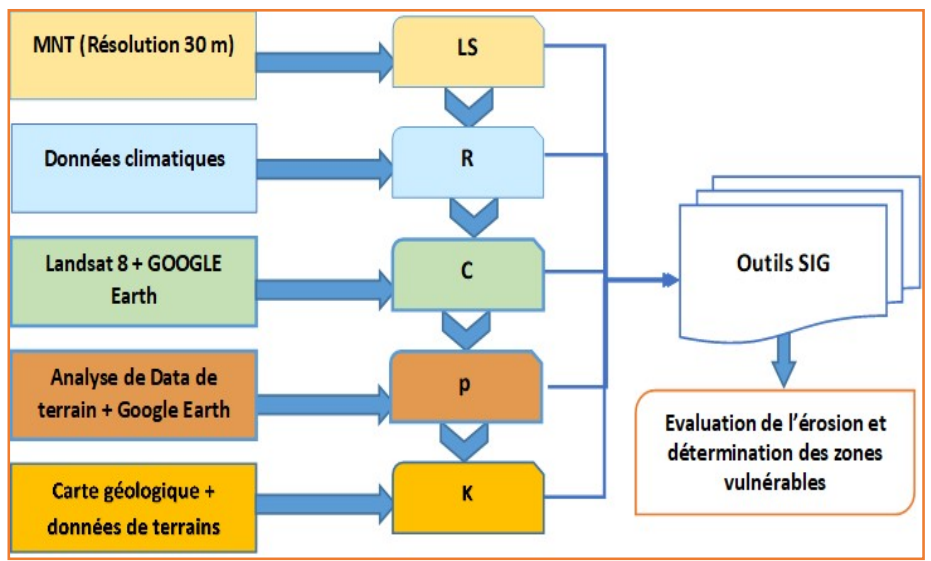

Figure 4. La méthodologie adoptée pour la réalisation de la carte des zones vulnérables au risque d'érosion hydrique dans le SBV d'Agoudal. 


\section{Description des facteurs d'érosion Facteur LS : Topographie}

Le facteur topographique (LS) est primordial dans le comportement érosif dans ces zones de hautes montagnes où la topographie devient déterminante dans les différentes phases du processus d'érosion, surtout dans la phase de transport des sédiments, ainsi que la longueur et l'inclinaison des pentes. Plus leurs valeurs augmentent, plus l'érosion est active sur les versants, et plus le ruissellement est élevé d'où les forts risques d'inondations.

L'étude topographique la zone d'étude a été réalisée en se basant sur les données les plus précises disponibles, notamment les cartes topographiques à l'échelle 1/50.000 (Feuilles: Outerbat, Imilchil Aghbalou n'kerdous et Aghendou N'Ouerz), qui ont été géoréférencées, numérisées. Sur la base du modèle numérique de terrain (MNT) de $30 \mathrm{~m}$ de résolution, téléchargé à partir du site web (https://earthexplorer.usgs.gov), le facteur LS est a été évalué en appliquant l'Equation(Eq.2) ci-dessous, développée par Mitasova et al. (1996) :

Où

$$
\mathrm{LS}=(\mathrm{L} / 22,13)^{\mathrm{m}} \cdot\left(65,41 \operatorname{Sin}^{2}(\mathrm{~S})+4,56 \operatorname{Sin}(\mathrm{S})+0,065\right)
$$

\footnotetext{
L: longueur de pente (en m)

$\mathrm{S}$ : inclinaison de la pente (en \%)

$\mathbf{m}$ : contant qui est égal à :
}

- $\quad 0,5$ pour les pentes supérieures à $\mathbf{5 \%}$

- $\quad 0,4$ pour les pentes de $\mathbf{3 , 5}$ à $\mathbf{5 \%}$

- $\mathbf{0 , 3}$ pour les pentes de $\mathbf{1}$ à $\mathbf{3 , 5 \%}$

- $\quad 0,2$ pour les pentes inférieures à $\mathbf{1 \%}$

Dans le cas des pentes les plus courantes ( 0,1 à $15 \%)$ d'une longueur de $60 \mathrm{~m}$, les valeurs de LS varient de 0,1 à 5 et dépassent 20 en zones de montagne où l'influence de l'inclinaison de la pente est déterminante. Les transports solides croissent de façon exponentielle (l'exposant $= \pm 1,4$ à 2) avec le pourcentage de pente (Zingg, 1940 ; Roose, 1996).

\section{Facteur d'érosivité pluviale $\mathbf{R}$}

L'énergie cinétique de la pluie contribue fortement à l'arrachement des particules solides. Elle est liée à l'intensité des pluies et dépend de la taille et de la vitesse de chute des gouttes. L'estimation du facteur de l'agressivité climatique selon la formule de Wischmeier et Smith (1978) nécessite la connaissance de l'énergie cinétique et l'intensité moyenne des pluies durant 30 minutes. Or, au niveau des stations climatiques de références du SBV d'Agoudal (Tableau 2), seules les données pluviométriques moyennes mensuelles et annuelles sont disponibles. Ainsi, l'équation appliquée (Eq.3) est celle développée par Rango \& Arnoldus (1987) qui se présente comme suit: 
Avec:

$$
\ln (R)=1,74 \log \Sigma \frac{p_{i}^{2}}{p} 1,29
$$

- $\quad \mathbf{p}_{\mathbf{i}}$ : précipitations mensuelles ;

- $\quad \mathbf{p}$ : précipitations annuelles ;

- $\quad \mathbf{R}$ : facteur de l'agressivité climatique.

Les caractéristiques climatiques des stations utilisées pour l'estimation des valeurs de l'érosivité de la zone d'étude sont les suivantes :

Tableau 1. Coordonnées des stations pluviométriques de référence

\begin{tabular}{|c|c|c|}
\hline \multirow{2}{*}{ Stations } & \multicolumn{2}{|c|}{ Coordonnées } \\
\cline { 2 - 3 } & $\mathbf{X}$ & $\mathbf{Y}$ \\
\hline M'semrir & 460759 & 122958 \\
\hline Imchil & 478855 & 173650 \\
\hline Tillouguit & 422674 & 158474 \\
\hline Zaouiet ahnsal & 433362 & 138594 \\
\hline
\end{tabular}

Tableau 2. Données pluviométriques des stations de référence

\begin{tabular}{|l|l|l|l|l|l|l|l|l|l|l|l|l|l|}
\hline Station & Janv. & Fév. & Mars & Avril & Mai & Juin & Juil & Aout & Sept & Oct. & Nov. & Déc. & $\begin{array}{l}\text { Cumul } \\
\text { Annuel }\end{array}$ \\
\hline M'semrir & 14,7 & 16,4 & 20,8 & 20,8 & 18 & 13,7 & 4,9 & 17,4 & 20 & 29,9 & 21,4 & 12,8 & 210,8 \\
\hline Imilchil & 1,9 & 8,4 & 23 & 30,8 & 42,1 & 29,9 & 17,3 & 22,8 & 60,3 & 78,6 & 31,5 & 31,5 & 377,9 \\
\hline Tillouguite & 46,6 & 47,5 & 51,5 & 37,1 & 23,7 & 9,7 & 3,5 & 11,5 & 14,7 & 23,8 & 42 & 39 & 350,5 \\
\hline $\begin{array}{l}\text { Zaouiet } \\
\text { Ahnsal }\end{array}$ & 36 & 34,2 & 39,9 & 39,2 & 25,6 & 23 & 6,6 & 23,2 & 26,2 & 38,2 & 35,3 & 35,5 & 362,9 \\
\hline
\end{tabular}

\section{Facteur du couvert végétal $C$}

Le facteur du couvert végétal $(\mathrm{C})$ est un paramètre clé dans la prédiction du taux d'activité érosive ; il est d'une grande utilité dans la protection contre l'érosion hydrique. Ses valeurs vont de 0.001 pour les sols bien couverts à 1 pour les sols nus (Wischmeier \& Smith 1978).

La carte d'occupation du sol a été élaborée à partir d'une image Landsat 8 prise le 28/3/2021, qui a été préalablement traitée, en utilisant l'indice de végétation normalisé NDVI (Normalized Difference Vegetation Index) permettant de distinguer les terrains nus de ceux boisés (Jensen, 2000). La réalisation d'une classification non supervisée, suivie d'une classification supervisée ont permis d’avoir la répartition de la couverture végétale dans la zone d'étude en se basant sur les compositions colorées des bandes de l'image (bandes 3, 4 et 5).

\section{Facteur des pratiques culturales $\mathbf{P}$}

C'est un paramètre qui permet de prendre en considération les aménagements antiérosifs existants dans l'estimation des pertes en sols des bassins versants. Chacune de ces mesures à un degré de protection dont le coefficient est établi suivant son efficacité à réduire le ruissellement et à 
atténuer les causes d'érosion (Benzougagh et al., 2020). Parmi les pratiques les plus efficaces de conservation des sols, on cite à titre indicatif les cultures en courbes de niveau, en bandes alternées ou en terrasses, les reboisements en banquettes, le buttage et le billonnage (Zouagui, 2018).

Les valeurs du facteur $P$ varient selon la pratique adoptée et aussi selon la pente. Ses valeurs sont inférieures ou égales à 1(Shin, 1999). Son maximum est attribué aux terrains sur lesquels aucune des pratiques citées n'est utilisée. Dnas le cas du bassin étudié dont les terrains sont majoritairement à vocation pastorale, où les pratiques culturales sont limitées aux petites parcelles sur les bords de l'oued selon nos prospections, on a attribué des valeurs approximatives à ce facteur selon le type d'occupation et les pratiques utilisées.

\section{Facteur érodibilité des sols $\mathrm{K}$ :}

L'interprétation des caractéristiques pédologiques de la zone cible a permis de les classer dans l'abaque de Wischmeier et Smith (1978) pour obtenir une approximation du facteur $\mathrm{K}$ en fonction des paramètres de la matière organique, de la structure, de la texture et de la perméabilité. La détermination de ces paramètres est difficile, faute de l'absence d'étude pédologique dans la zone d'étude.

Le facteur $\mathrm{K}$ est déterminant dans l'équation de Wischmeier et Smith (1978) (Eq.4) du moment qu'il reflète le degré d'érodibilité des sols à travers la détermination de leur composition. Ainsi son estimation s'est basée sur l'analyse des échantillons des sols prélevés dans la zone d'étude en appliquant l'équation (Eq.4 ) suivante:

$$
100 \mathrm{~K}=2,1 \mathrm{M}^{1,14} \cdot 10^{-4}(12-\mathrm{a})+3,25(\mathrm{~b}-2)+2,5(\mathrm{C}-3)
$$

\section{Avec :}

K : Érodibilité des sols en ha.H/ha.MJ.mm ;

M : (\% Sable fin +\% Limon) *(100-\%argile);

a : \% de la matière organique ;

b : code de la structure de sol (1 à 4$)$ :

1 : Très fine $; 2:$ fine $; 3:$ moyenne et grossière $; \mathbf{4}:$ très grossière

c : code de perméabilité ( $\mathbf{1}$ à $\mathbf{6}): \mathbf{1}$ rapide ; $\mathbf{2}$ moyenne à rapide ; $\mathbf{3}$ modérée ; $\mathbf{4}$ lente à modérée ; 5 lente et $\mathbf{6}$ très lente.

Les valeurs du facteur K sont ajustées dans la zone d'étude et ont été obtenues en utilisant l'équation (Eq.5) suivante :

$$
K_{\text {ajusté }}=\text { K. }\left(0,983-0,0189 X+0,0000973 X^{2}\right)
$$

Avec :

K : Facteur d'érodibilité de Wischmeier ;

$\mathbf{X}$ : le pourcentage des éléments grossiers de surface ayant une taille de plus de $2 \mathrm{~mm}$ 
L'utilisation de cette formule(Eq.5) a nécessité l'évaluation des différents facteurs de l'équation universelle sur l'ensemble de la zone d'étude et leur expression sous forme de cartes thématiques. Ces cartes ont été ensuite intégrées dans un système d'information géographique(SIG) libre, le Quantum GIS (QGIS).

\section{Calcul de la dégradation spécifique des sols}

Pour évaluer la part produite et acheminée à l'exutoire, le modèle RUSLE utilisé ne permet d'estimer que l'érosion produite sur les versants. Pour ce faire, il est impératif de prendre en compte le facteur de correction qui est fonction de la dénivelée du sous-bassin et de la longueur du cours d'eau principal. Ce coefficient (ou delivery ratio) a été défini par Hession et Shanholz (1988) selon l'équation (Eq.6) suivante

$\mathrm{DR}=\mathbf{1 0}$. $\mathrm{D} / \mathrm{L}$

Où

DR : Coefficient de transport ; D : Dénivelée du BV ; L : Longueur du Thalweg principal).

\section{Résultats et discussion \\ Résultats des facteurs}

Facteur R

Après le calcul du facteur $\mathrm{R}$ au niveau des stations de référence selon la formule de Rango et Arnoldus (1989). Une interpolation a été effectuée à l'aide de l'outil SIG en vue de spatialiser ce facteur sur l'ensemble du SBV d'Agoudal. Les résultats obtenus sont résumés dans le Tableau 3 et illustrés par la Figure 5 ci-dessous :

Tableau 3. Calcule du facteur $\mathrm{R}$ dans les stations de référence

\begin{tabular}{|c|c|c|c|c|}
\hline \multirow{2}{*}{ Station } & \multicolumn{2}{|c|}{ Coordonnées } & \multirow{2}{*}{$\begin{array}{c}\text { Précipitations } \\
\text { moyennes Annuelles }\end{array}$} & $\begin{array}{c}\text { Indice } \\
\text { d'érosivité }\end{array}$ \\
\cline { 2 - 3 } & $\mathrm{X}$ & $\mathrm{Y}$ & 210,8 & 34,25 \\
\hline M'semrir & 460759 & 122958 & 377,9 & 65,06 \\
\hline Imchil & 478855 & 173650 & 350,5 & 56,44 \\
\hline Tillouguit & 422674 & 158474 & 362,9 & 50,94 \\
\hline Zaouiet ahnsal & 433362 & 138594 & \\
\hline
\end{tabular}

L’érosivité pluviale affiche des valeurs plus élevées dans les stations en altitude. A Imilchil, l'agressivité enregistre une valeur de l'ordre de 65 (Tableau 3) qui est relativement élevée par rapport aux stations de Zaouiet Ahensal et Tillouguite, et encore plus élevée que celle de la station de M'semrir qui ne dépasse guère 34 . 


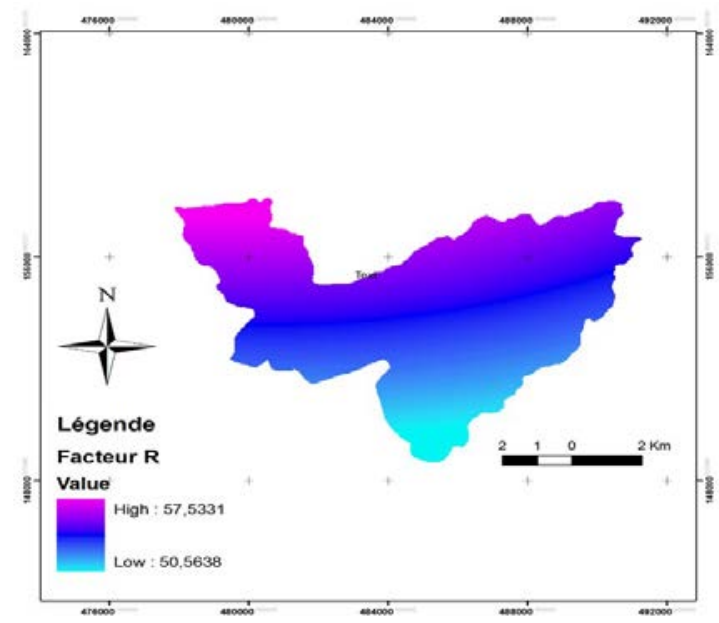

Figure 5. Carte du facteur R de la zone d'étude

Les pluies dans la zone d'étude sont caractérisées par leur caractère torrentiel. Elles sont mal réparties et très concentrées notamment en septembre et octobre (39\% des pluies annuelles), au début de la saison culturale lorsque les sols cultivés sont peu couverts, et la végétation spontanée très médiocre.

L'agressivité pluviale ne présente pas une grande variabilité au niveau du $\mathrm{SBV}$, en on distingue deux classes (Figure 6). La première (40-50) couvre moins de $19 \%$ et la deuxième (50-60) domine sur plus de $80 \%$ de la zone d'étude. Les fortes valeurs de ce facteur se présentent principalement sur les versants exposés au nord de cette vallée de montagne dont les précipitations sont majoritairement sous forme d'averses orageuses surtout en automne, ce qui prévisualise son rôle majeur dans la génération des processus érosifs.

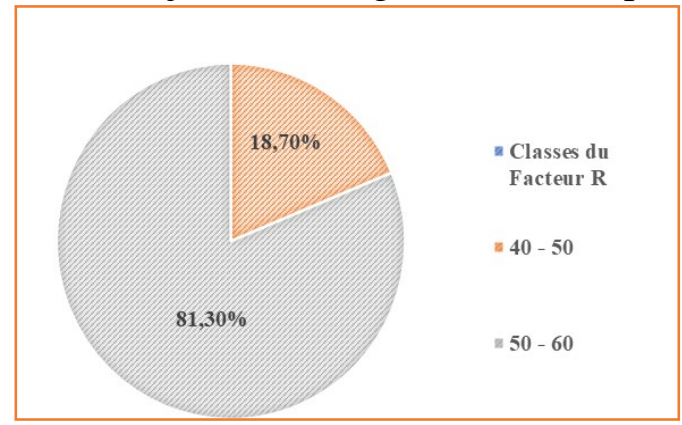

Figure 6. Répartition des classes du facteur d'érosivité pluviale R

\section{Facteur LS}

Dans la zone d'étude, les pentes sont fortes et peu longues, en raison des formes de plissement des reliefs, avec alternance de couches dures et tendres qui dominent le paysage du SBV. Les valeurs du facteur LS varient entre 2,7 et 200 comme valeur maximale observée, avec une moyenne de 
l'ordre d'environ $40(39,900)$ (Tableau 4), les autres caractéristiques sont relatées ci-dessous :

Tableau 4. Caractéristiques du facteur topographique dans la zone d'étude

\begin{tabular}{|c|c|}
\hline Paramètres & Valeurs \\
\hline Nombre d'unités & 27626 \\
\hline Valeur minimale & 2.697 \\
\hline Valeur maximale & 199.871 \\
\hline Valeur moyenne & 39.900 \\
\hline Ecart type & 36.637 \\
\hline
\end{tabular}

La spatialisation de ce facteur montre que la grande partie du SBV étudié affiche des valeurs de près de 40 (Figure 7), alors que celles très hautes sont marquées sur des versants abrupts, où l'inclinaison dépasse de loin $45 \%$.

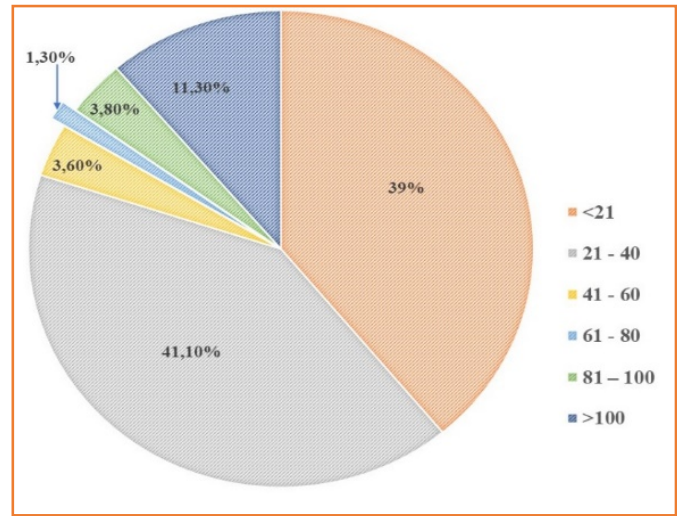

Figure 7. Ventilation des classes du facteur topographique (LS)

L'analyse des classes de pente (Figure 8) montre que les pentes très fortes de plus de $45 \%$ sont observées sur les reliefs abrupts et les falaises présents dans plusieurs endroits de ce SBV où $27 \%$ de sa surface totale est incluse dans cette tranche. la classe de moins de $5 \%$ est faiblement représentée et correspond au zone des bas-fonds et talwegs.

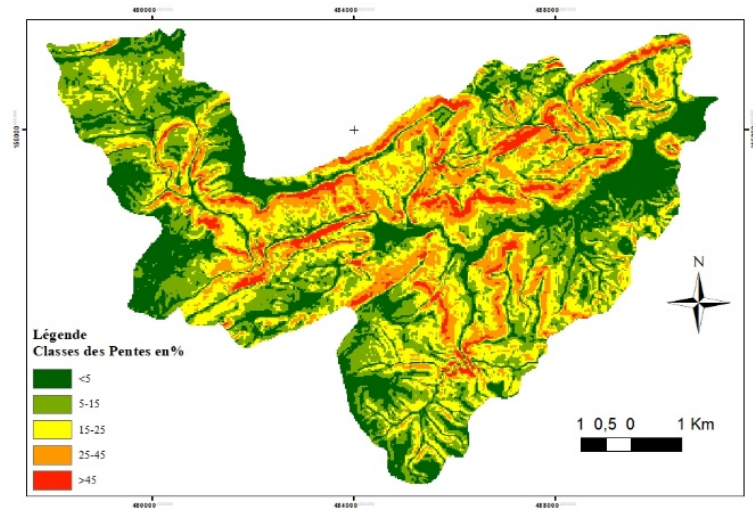

Figure 8. Classes des Pentes (en\%) de la zone d'étude 


\section{Facteur C : Couverture végétale}

La combinaison de l'occupation du sol et du taux de recouvrement dans la zone d'étude, avec les caractéristiques des pratiques culturales, nous a permis d'établir les caractéristiques du facteur C (Tableau 5, Tableau 6, Figure 9, Figure 10):

Tableau 5. Caractéristiques du facteur du couvert végétal C de la zone d'étude

\begin{tabular}{|l|l|}
\hline Paramètre & Valeurs du facteur C \\
\hline Valeur minimale & 0,25 \\
\hline Valeur maximale & 1 \\
\hline Valeur moyenne & $\mathbf{0 , 6 5}$ \\
\hline Ecart type & 0,3 \\
\hline
\end{tabular}

Ces chiffres (Tableau 5) révèlent que la moyenne du facteur $C$ est assez élevée (0.65). Ceci est dû à la faible protection des sols par le couvert végétal et à la dégradation des terrains pastoraux.

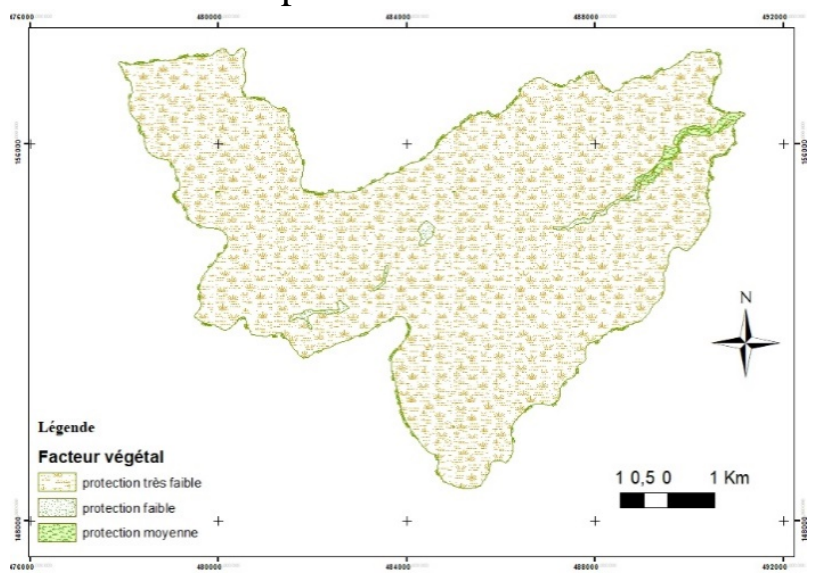

Figure 9. Carte du facteur C de la zone d'étude

La classe des parcours à très faible couverture annuelle et à faible densité s'étend sur plus de $70 \%$ de la zone d'étude, ce qui se traduit par la faible protection du sol contre l'érosion hydrique (Tableau 6).

Tableau 6. Répartition des classes d'occupation du sol et facteur C

\begin{tabular}{|c|c|c|}
\hline Types d'occupation du sol & $\begin{array}{c}\text { Indice du } \\
\text { facteur C }\end{array}$ & $\begin{array}{c}\text { Superfi } \\
\text { cie (\%) }\end{array}$ \\
\hline Constructions & 1 & 0 \\
\hline Parcours à couverture végétale à base de xérophytes & 0,8 & 26,9 \\
\hline Parcours de lit d'oued à très faible recouvrement du sol & 0,95 & 0,9 \\
\hline Parcours à très faible couverture annuelle à faible densité & 0,9 & 70,8 \\
\hline Terrains agricoles irrigués de bonne couverture du sol & 0,25 & 0,8 \\
\hline Terrains agricoles irrigués de couverture moyenne semestrielle & 0,35 & 0,6 \\
\hline
\end{tabular}

En second lieu, on trouve la classe dont la couverture végétale à base de xérophytes qui occupe environ $27 \%$. Seule une petite superficie de l'ordre 
de 1.4\% du SBV d'Agoudal qui est dotée d'une couverture végétale moyenne à bonne avec des valeurs du facteur $C$ de moins de 0,25 à 0,3 représentées par les deux dernières classes qui correspondent sur le terrain aux petites parcelles agricoles au bord du lit de l'Oued d'Assif Melloul (Tableau 6).

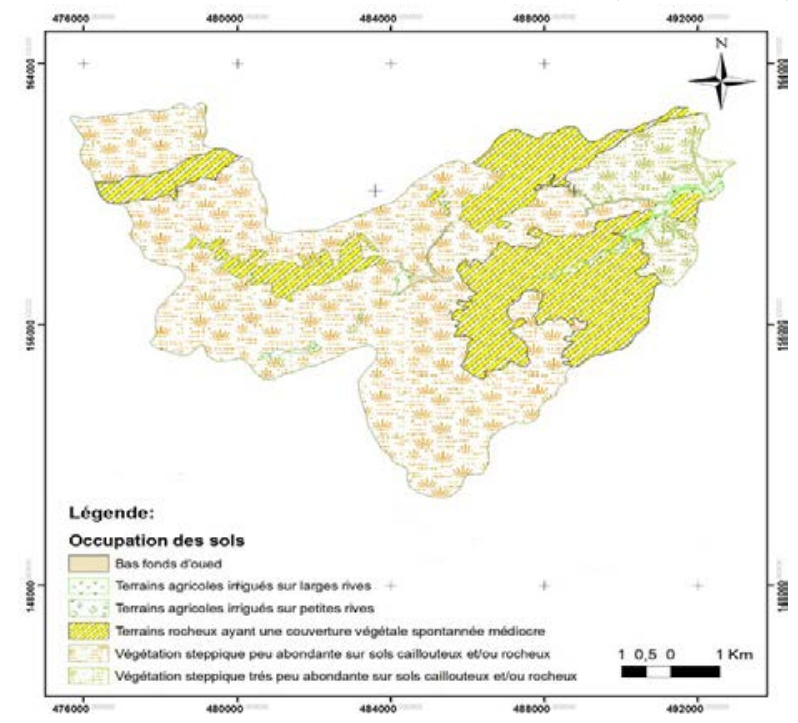

Figure 10. Carte d'occupation des sols du SBV d'Agoudal

Les steppes de xérophytes abondantes en nappes par endroit dans la zone d'étude, elles sont caractérisées par leur partie aérienne annuelle, mais leur appareil racinaire, assez développé et pérenne, pourrait logiquement constituer une armature pour le substrat et fortifier sa résistance vis-à-vis de l'érosion hydrique. Cependant ces formations steppiques sont elles aussi victimes de déboisement massif par les populations locales pour répondre à leurs principaux besoins journaliers : cuisson, chauffage d'eau de douche et le chauffage d'habitat en période froide. Ce qui se répercute négativement sur la couverture et la protection des sols déjà peu couverts.

\section{Facteur $\mathbf{P}$ : pratiques culturales}

Ce facteur permet de prendre en considération les aménagements antiérosifs existants relatifs à l'estimation des pertes en sols. Chaque mesure a un degré de protection dont le coefficient est établi suivant son efficacité à réduire ou à atténuer les sources d'érosion.

Les résultats de ce facteur montrent que les valeurs de $P$ variant entre 0,7 et 0,9 correspondant aux terrains assez bien à peu protégés. ces derniers couvrent moins de 1,5\% de la superficie du SBV étudié, alors que plus de 98\% de ces terrains dont la valeur de P est égale à 1 (Shin, 1999) sont sans mesures protectrices (Tableau 7) 
Tableau 7. Répartition des classes du facteur P dans le SBV d'Agoudal

\begin{tabular}{|c|c|c|}
\hline & Indice de P & Superficie (\%) \\
\hline Pratiques assez bien protectrices & 0,7 & 0,8 \\
\hline Pratiques peu protectrices & 0,9 & 0,6 \\
\hline Absence de pratiques protectrices & 1 & $\mathbf{9 8 , 6}$ \\
\hline
\end{tabular}

\section{Facteur k : érodibilité des sols}

Les données préliminaires des caractéristiques d'érodibilité des sols du SBV d'Agoudal (Tableau 8) sont obtenus en appliquant l'équation de Wischmeier et Smith (1978).

Tableau 8. Caractéristiques du facteur d'érodibilité des sols

\begin{tabular}{|c|c|}
\hline & Valeurs de K \\
\hline Valeur minimale & 0,1400 \\
\hline Valeur maximale & 0,4576 \\
\hline Valeur moyenne & $\mathbf{0 , 3 9 7 0}$ \\
\hline Ecart type & 0,0793 \\
\hline
\end{tabular}

Les valeurs préliminaires d'érodibilité (Figure 11) révèlent que la classe $\mathrm{K}$ de 0.36 à 0.4 qui intègrent les sols érodibles représente $80 \%$ de la superficie du SBV d'Agoudal avec une moyenne de plus de $\mathbf{0 , 3 9}$ (Tableau 8).

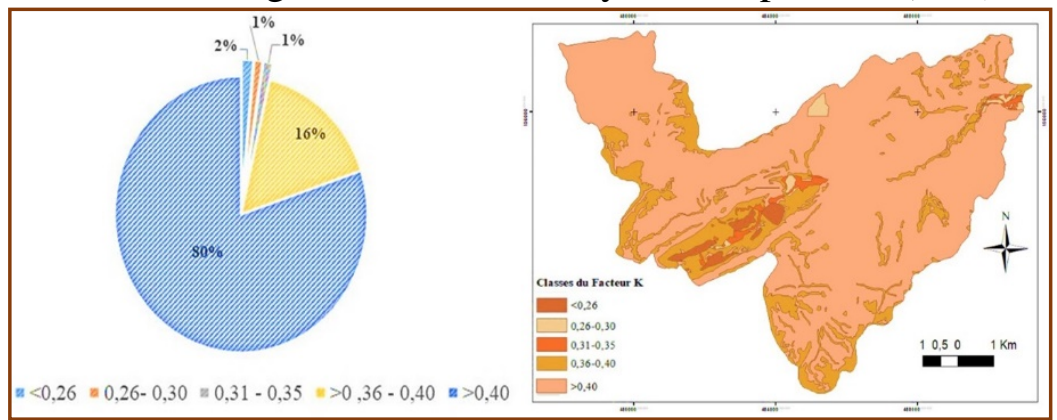

Figure 11. Carte et proportion des classes du facteur K dans le SBV d'Agoudal

Lors des prospections de terrains, il a été remarqué la présence des éléments grossiers où sont observés plusieurs affleurements rocheux sur les versants et surtout sur les sommets calcaires et marno-calcaires qui sont charriés avec l'avancement des processus érosifs vers les mi et bas-versants et deviennent ainsi les éléments prépondérants en surface du SBV. Pour cela nous avons procédé à l'ajustement de la valeur du facteur $\mathrm{K}$ en l'Equation (Eq.5) sus-indiquée.

Les classes du facteur d'érodibilité ajustée représentant la friabilité la plus élevé qui est $>0,2$ couvre $8 \%$ de la superficie du SBV, cédant la place aux classes moins érodibles (Figure 12). Ces changements des taux révélés après ajustement de $\mathrm{K}$, s'expliquent par la dominance des éléments grossiers 
couvrant la majorité du paysage de la zone d'étude, ce qui est en adéquation avec les prospections de terrain et les analyses granulométriques des sols.

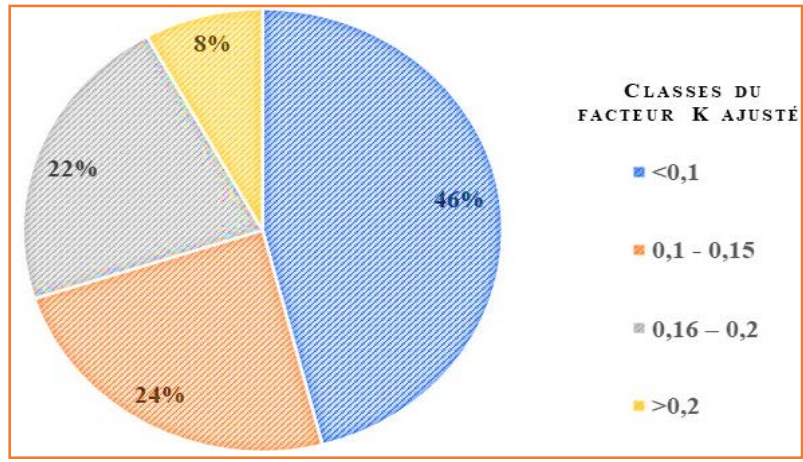

Figure 12. Répartition des classes du facteur K ajusté

\section{Évaluation des pertes en sols}

Le modèle empirique de Wischmeier et Smith (1978) présente plusieurs limites d'adaptation sous les conditions différentes de celles où il a été élaboré. Il considère que toutes les surfaces sont soumises à l'érosion, si aucun des facteurs n'est nul, et ne met pas en évidence les dépôts aussi bien de versants que fluviatiles.

Les résultats de l'application de ce modèle après modélisation des cinq facteurs en cartes thématiques, via SIG, ont permis l'évaluation des facteurs causaux et leur spatialisation sur l'ensemble du SBV étudié. La valeur moyenne d'érosion au champ obtenue est $\mathbf{2 5 5 , 0 5 8}$ t/an/ha avec un écart type d’un peu moins de 285 t/an/ha (Tableau 9), en réponse à l’hétérogénéité du milieu et de ses conditions.

Tableau 9. Caractéristiques des classes d'érosion au champ dans le SBV d’Agoudal

\begin{tabular}{|c|c|}
\hline & Valeurs en t/ha/an \\
\hline Valeur minimale & 1,223 \\
\hline Valeur maximale & 1987,730 \\
\hline Valeur moyenne & $\mathbf{2 5 5 , 0 5 8}$ \\
\hline Médiane & 154,680 \\
\hline Ecart type & 284,967 \\
\hline
\end{tabular}

Ces valeurs paraissent, au premier regard, un peu élevées, mais la réalité montre que ces processus sont d'autant plus actifs que les sols ont subi des changements profonds dans leurs structures, qui ont induit des dérapages généralisés avec le temps. D'où l'affleurement de la roche mère sur la quasitotalité des versants du SBV, où de grands terrains nus décapés de sols partout abondants avec affleurement des roches squelettiques, y compris les ravines, et qui témoignent de l'évolution rapide de ce fléau dans ces écosystèmes très vulnérables où tous les facteurs sont conjugués pour accélérer les taux d'érosion. 
Les données relatées ci-après (Tableau 10), montrent que les classes 50 à $400 \mathrm{t} / \mathrm{ha} / \mathrm{an}$ sont les plus représentées et couvrent environ $66 \%$ soit 2/3 de la zone d'étude, avec un taux d'érosion très élevé d'une valeur maximale de 1987,73 t/ha/an, et valeur moyenne d'environ $255 \mathrm{t} / \mathrm{ha} / \mathrm{an}$, avec un écart type d'environ 285 t/ha/an (Tableau 9). Un tel taux est loin d'être tolérée par ses sols qui subissent, en plus de la carence en végétaux, un climat agressif avec des précipitations à caractère orageux très localisées dans le temps et l'espace.

Tableau 10. Classes de l'érosion au champ dans le SBV d'Agoudal

\begin{tabular}{|c|c|c|}
\hline $\begin{array}{c}\text { Degré d'érosion par } \\
\text { versant(t/ha/an) }\end{array}$ & Superficie (\%) & Superficie cumulées(\%) \\
\hline$<50$ & 18,1 & 18,1 \\
\hline $50-100$ & 20,2 & 38,3 \\
\hline $101-150$ & 14,8 & 53,1 \\
\hline $151-200$ & 11 & 64,1 \\
\hline $201-300$ & 11,7 & 75,8 \\
\hline $301-400$ & 7,8 & 83,6 \\
\hline $401-500$ & 4,5 & 88,1 \\
\hline $501-1000$ & 7,1 & 95,2 \\
\hline$>1000$ & 4,8 & 100 \\
\hline
\end{tabular}

Les chiffres ainsi constatés dépassent de loin ce que la pédogenèse peut produire pour compenser ces pertes dont les conséquences se traduisent par une baisse des rendements des cultures et une réduction de la superficie des Surface Agricoles Utiles (SAU) concentrés sur les berges des oueds.

Ces résultats sont généralement plus élevés que ceux signalés dans des recherches antérieures dans d'autres régions du Haut Atlas, où les classes de perte en sol y sont comprises entre 50 et 400 t/ha/an, couvrent environ la moitié des zones étudiées. Citons à juste titre les cas d'Oum Er-Rabia et d'Ourika dans lesquels ces classes touchent respectivement $54 \%$ dans le premier cas (Yjjou et al., 2014) et 48 \% dans le deuxième (Meliho, et al., 2016).

\section{Dégradation spécifique}

Les résultats obtenus après correction (Tableau 11 et Figure 13) correspondent à la dégradation spécifique du SBV qui montrent que la majorité de sa superficie est soumise à une dégradation très forte, au-delà du seuil de tolérance de sols.

Tableau 11. Taux d'érosion au champ dans le SBV d'Agoudal

\begin{tabular}{|c|c|c|c|c|c|c|}
\hline \multirow[t]{2}{*}{$L(m)$} & \multirow[t]{2}{*}{$D(m)$} & \multirow[t]{2}{*}{ DR } & \multicolumn{3}{|c|}{ Dégradation spécifique(t/h/an) } & \multirow{2}{*}{$\begin{array}{l}\text { Perte } \\
\text { totale(t/an) }\end{array}$} \\
\hline & & & Min & $\operatorname{Max}$ & Moyenne & \\
\hline 18485 & 700 & 0,38 & 0,46 & 755,3 & 96,9 & 605528 \\
\hline
\end{tabular}




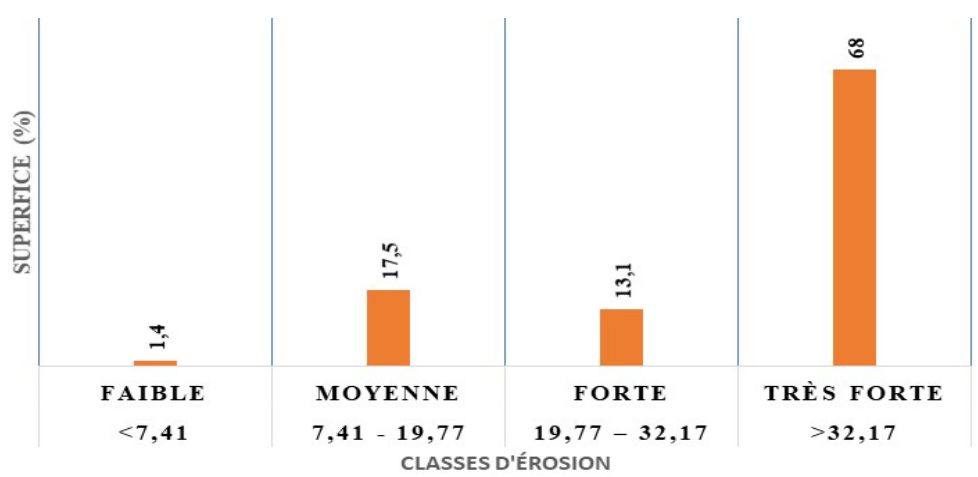

Figure 13. Importance et degré de la dégradation spécifique dans la zone d’étude

On remarque que moins de $1,5 \%$ de la superficie de la zone d'étude est soumise à des taux inférieurs à 7,4 t/ha/an, alors que seulement $18.9 \%$ de la superficie globale du SBV affiche des taux d'érosion de degré faible et moyen. Ces superficies correspondent aux zones qui allongent les cours d'eaux principaux et correspondent aux bas-versants situés sur des pentes de moins de $15 \%$ avec la présence remarquable d'éléments grossiers offrant une certaine protection qui amoindris l'effet des gouttes de pluies et atténuer l'énergie cinétique du ruissèlement.

L'impact de chacun des facteurs entrant dans l'équation de l'érosion agit différemment. En effet, le facteur topographique et celui d'érodibilité, dans cette zone, sont deux variables importantes dans la sensibilité à l'érosion, vue que les taux de dégradation des sols leur sont proportionnels. En revanche la longueur et l'inclinaison de pente ne sont pas de bons indicateurs, vue que les taux d'érosion les plus élevés ont été enregistrés au niveau des classes LS entre 15 et $25 \%$.

Après avoir analysé les différents facteurs causaux de l'érosion dont les résultats ont été confrontés aux données officielles recueillies (cartes géologiques et topographiques de la zone d'études, données climatiques, les études sur l'érosion réalisées par les départements étatiques concernés) et aux réalités de terrain, nous avons procédé à la cartographie des zones vulnérables vis-à-vis des processus d'érosion et de dégradation des sols (Figure 14). Un tel résultat pourrait à notre sens servir pour les différents opérateurs publics concernés, les acteurs de la socièté civile et toutes les parties prenantes impliquées dans le processus de lutte antiérosive surtout dans la planification des opérations de protection du sol et des ressources microbilogiques, rhizosphériques et pédologiques dans cette zone de montagne très nécessiteuse. 


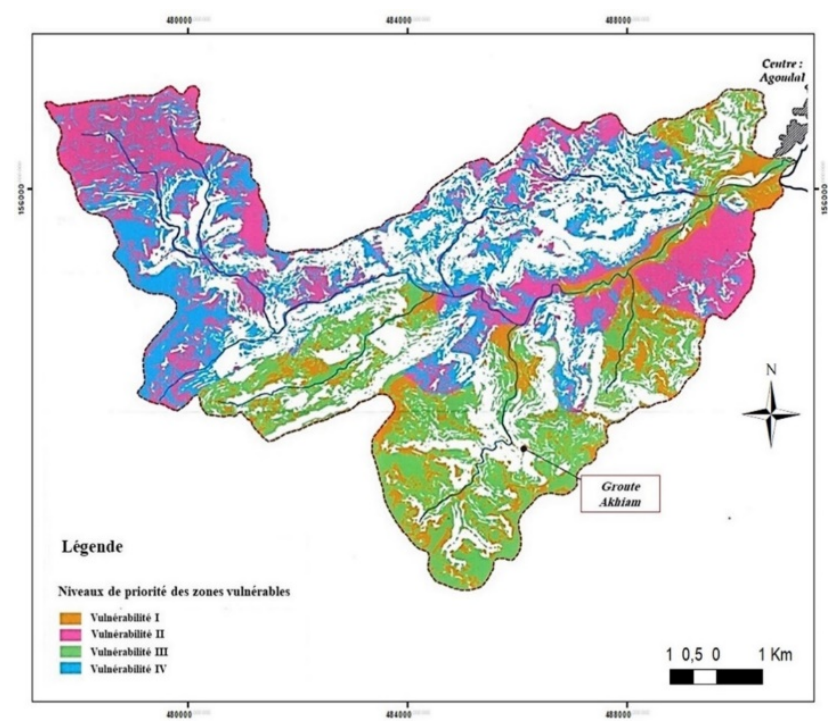

Figure 14. Carte des zones vulnérables et leur niveau de priorité dans le SBV d’Agoudal

Quatre niveaux de vulnérabilité ont été identifiés et spatialisés en vue de visualiser les sites prioritaires aux risques de dégradation pouvant servir en cas d'éventuelles interventions dans le cadre des programmes d'aménagement du SBV d'Agoudal en amont de la vallée d'Imilchil.

L'identification de ces zones a été faite sur des critères basés essentiellement sur le taux moyen d'érosion de 255,058 t/ha/an (Tableau 9) et les classes de dégradation spécifique. Sachant bien que $68 \%$ de la superficie totale du SBV d'Agoudal est soumise à une dégradation supérieure à 32,7\% et que seulement $1,4 \%$ de la superficie affiche des taux inférieurs à 7,4\% (Figure 13). Cette priorisation a été faite pour cadrer les zones vulnérables et potentiellement aménageables dans le but de réduire l'érosion et la génération des crues, et de protéger les agglomérations et infrastructures avales.

\section{Conclusion}

Les résultats obtenus dans le cadre du présent travail, affirment que la zone d'étude est menacée par le phénome de l'érosion hydrique et de la dégradation continue de la qualité des sols dont les conséquences impactent négativement le développement durable de cette région d'une part. D'autre part ces résultats revèlent l'intérêt de la télédétection et du SIG dans l'évaluation de la vulnérabilité à l'érosion dans ces zones de haute montagne asylvatique qui est sans doute d'une grande utilité dans la recherche des solutions adéquates.

La méthode utilisée a permis de montrer que le SBV étudié est soumis aux menaces de l'érosion hydrique dont le taux moyen dépasse $255 \mathrm{t} / \mathrm{ha}$ /an avec des taux de dégradation spécifique supérieurs à 7,41 t/ha/an touchant plus de 
$98 \%$ de sa superficie totale dont $68 \%$ enregistre une dégradation spécifique très élevée au-delà de 32,17t/ha/an.

L'exploitation de ces résultats nous a conduit à identifier et à priosriser les zones à risque dans l'Atlas central, en l'occurence le SBV d'Agoudal en amont du BV d'Oum Er-Rbia. Ce qui peut servir de modèle pouvant être généralisé sur le SBV d'Assif Melloul qui constitue sa continuité avale et qui souffre de la même problématique. Comme il peut être transposable à d'autres régions du haut Atlas central, en raison des similitudes de leurs caractéristiques géomorphologiques, géoloegiques, pédologiques, édaphiques et climatiques.

Ainsi, la carte de vulnérabilité à l'érosion synthétisée, pourrait constituer un document de base pour tous les programmes et les actions futurs d'aménagement et de gestion prévus pour atténuer ce fléau et ses conséquences néfastes sur le fonctionnement des écosystèmes agropasotoraux de la région.

\section{Remerciements}

$\mathrm{Au}$ terme de ce travail, nous tenons à présenter nos vifs sentiments de reconnaissance et nos remerciements à tous ce qui ont collaboré de près et de loin à l'accomplissement des différentes phases de cette recherche. Citons à ce propos, entre autres :

- Monsieur le doyen de la faculté des sciences de Meknès et ses collaborateurs ;

- L’Association Akhiam et tous ses partenaires mobilisés dans le processus de lutte antiérosive dans la zone d'étude (notamment la FAO, le PNUD, le GEF, l'ORMVAT, Services des Eaux et Forêts, l'Agence du Bassin Hydraulique d'Oum Er-Rabia, les communautés villageoises «Jmaâ), l'ONG française SENS, Hydraulique Sans Frontières, etc.)

\section{References:}

1. Amrhar, M. (1995). Tectonique et inversions géodynamiques post-rift dans le Haut-Atlas occidental: structures, instabilités tectoniques et magmatismes liés à l'ouverture de l'Atlantique central et la collision Afrique-Europe. Doctorat thesis, University of Marrakech, Morocco, 253.

2. Anys, H., Bonn, F., \& Merzouk, A. (1994). Remote sensing and gis based mapping and modeling of water erosion and sediment yield in a semi-arid watershed of Morocco. Geocarto International, 9(1), 31-40.

3. Anys, H., Bonn, F., \& Merzouk, A. (1992). Cartographie et calcul de l'érosion hydrique a l'aide de télédétection et du SIG, cas du bassin versant d'oued Aricha(Settat, Maroc), Géo Observateur, 2:37-51. 
4. Badraoui, A. \& Hajji, A. (2001). "Envasement des retenues de barrages." La Houille Blanche(6-7): 72-75.

5. Benbrahim, K. F., Ismaili, M., Benbrahim, S. F., \& Tribak, A. (2004). Problèmes de dégradation de l'environnement par la désertification et la déforestation : impact du phénomène au Maroc. Science et changements planétaires/Sécheresse, 15(4), 307-320.

6. Bennmoussa, M., Elyamani, M., \& Saidi, A. (1993). Application de l'équation universelle des pertes en sols au bassin versant d'Oued Tasrafate, Géo Observateur, 3 :83-93.

7. Benzougagh, B., Meshram, SG., Dridri, A., Boudad, L., Sadkaoui, D., Mimich, K., \& Khedher, KM. (2020). Mapping of soil sensitivity to water erosion by RUSLE model: case of the Inaouene watershed (Northeast Morocco). Arabian Journal of Geosciences (2020) 13:1153.

8. Boudhar, A., Duchemin, B., Hanich, L., Chaponnière, A., Maisongrande, P., Boulet, G. \& Chehbouni, A. (2007). Analyse de la dynamique des surfaces enneigées du Haut Atlas marocain à partir des données SPOT-VEGETATION. Science et changements planétaires/Sécheresse, 18(4), 278-288.

9. Charrière, A., \& Haddoumi, H. (2016). Les «Couches rouges» continentales jurassico-crétacées des Atlas marocains (Moyen Atlas, Haut Atlas central et oriental): bilan stratigraphique, paléogéographies successives et cadre géodynamique. Boletín geológico y minero, 127(2-3), 407-430.

10. Charrière, A., Ibouh, H., \& Haddoumi, H. (2011). Le Haut Atlas central de Beni Mellal à Imilchil. Nouveaux Guides géologiques et miniers du Maroc, 4, 109-164.

11. Charrière, A., Haddoumi, H., Mojon, P. O., Ferrière, J., Cuche, D., \& Zili, L. (2009). Mise en évidence par charophytes et ostracodes de l'âge Paléocène des dépôts discordants sur les rides anticlinales de la région d'Imilchil (Haut Atlas, Maroc): conséquences paléogéographiques et structurales. Comptes Rendus Palevol, 8(1), 919.

12. Choubert, G. \& Faure-Muret, A. (1960). "Evolution du domaine atlasique marocain depuis les temps paléozoïques." Mémoire horssérie-Société géologique de France(1): 447-527.

13. Croitoru, L. \& Sarraf, M. (2017). Le Coût de la Dégradation de l'Environnement au Maroc. Environment and Natural Resources Global Practice Discussion Paper, vol. 5.

14. Elalaoui, A., Marrakchi, C., Fekri, A., Maimouni, S., \& Aradi, M. (2015). Mise en place d'un modèle qualitatif pour la cartographie des zones à risque d'érosion hydrique dans la chaîne atlasique: cas du 
bassin versant de la Tessaoute amont. (Haut Atlas Central, Maroc). Eur Sci J 11(29): 106-121.

15. El Garouani, A., Chen, H., Lewis, L., Tribak, A., \& Abharour, M. (2008) Cartographie de l'utilisation du sol et de l'érosion nette à partir d'images satellitaires et du sig idrisi au nord-est du maroc. Télédétection 8(3):193-201.

16. El Garouani, A., Merzouk, A., Jabrane, R., \& Boussema, MR. (2005) Analyse spatiale de l'érosion en nappe et de l'infiltrabilité des sols dans le PréRif marocain. Télédétection 5(1-2-3): 69-80

17. El Garouani, A., Merzouk, A., \& Ozer, A. (2003). Cartographie et évaluation quantitative de l'érosion hydrique au Prérif marocain. IAHS Publication, Wallingford, pp 380-388

18. Froitzheim, N., Stets, J., \& Wurster, P. (1988). Aspects of western High Atlas tectonics. In The Atlas System of Morocco (pp. 219-244). Springer, Berlin, Heidelberg.

19. Ghanam, M. (2003). La désertification au Maroc-Quelle stratégie de lutte. In 2nd FIG Regional Conference Marrakech (pp. 2-5).

20. Hession, W.C. \& Shanholtz, V. O. A. (1988). Geographic information system for targeting nonpoint-source agricultural pollution. Journal of Soil and Water conservation, vol. 43, no 3, p. 264-266.

21. Houbert, G. \& "Faure Muret, A. (1962). Évolution du domaine atlantique marocain depuis les temps paléozoïques." Mém. hs Soc. géol. France, Livre mémoire P. Fallot 1: 447-527.

22. Ibouh, H., Michard, A., Charrière, A., Benkaddour, A., \& Rhoujjati, A. (2014). Tectonic-karstic origin of the alleged "impact crater" of Lake Isli (Imilchil district, High Atlas, Morocco). Comptes Rendus Geosciences 346:82-89.

23. Ibouh, H. \& Chafiki, D. (2017). La tectonique de l'Atlas: ageet modalit es. Le Maroc, paradis des g eologues: Soci et eg eologue de France 194.

24. Issa, LK., Lech-Hab, KBH., Raissouni, A., \& El Arrim, A. (2016) Cartographie quantitative du risque d'erosion des sols par approche SIG/USLE au niveau du bassin versant Kalaya (Maroc Nord Occidental). J Mater Environ Sci 7(8): 2778-2795.

25. Jenny, J., Le Marrec, A., \& Monbaron, M. (1981). Les couches rouges du Jurassique moyen du Haut Atlas central (Maroc); Correlations lithostratigraphiques, elements de datations et cadre tectonosedimentaire. Bulletin de la Société géologique de France, 7(6), 627640.

26. Jensen, J. R. (2000). Remote Sensing of the Environment An Earth Resource Perspective Prentice Hall. Upper Saddle River (NJ), USA. 
27. Laville, E., Lesage, J. L., \& Seguret, M. (1977). Geometrie, cinematique (dynamique) de la tectonique atlasique sur le versant sud du Haut Atlas marocain; apercu sur les tectoniques hercyniennes et tardi-hercyniennes. Bulletin de la Société géologique de France, 7(3), 527-539.

28. Maimouni, S., et al. (2011). "Potentiels et limites des indices spectraux pour caractériser la dégradation des sols en milieu semi-aride." Canadian Journal of Remote Sensing 37(3): 285-301.

29. Meliho, M., et al. (2016). "Cartographie Des Risques De L'érosion Hydrique Par L'équation Universelle Révisée Des Pertes En Sols, La Télédétection Et Les SIG Dans Le Bassin Versant De L’Ourika (Haut Atlas, Maroc)." Eur Sci J 12(32): 227-297.

30. Merkhi, A., Laftouhi, NE., Soulaimani, A., \& Fniguire, F. (2015). Quantification et évaluation de l'érosion hydrique en utilisant le modèle RUSLE et déposition intégrés dans un SIG. Application dans le bassin versant n'fis dans le haut atlas de Marrakech (Maroc). Eur Sci J 11(29):340- 356.

31. Michard, A., Ibouh, H., \& Charri ere, A. (2011). Synclinetopped anticlinal ridges (STARs) from the High Atlas: a Moroccan conundrum, and inspiring structures from the Syrian Arc, Israel. Terra Nova. 23:314-323.

32. Mitasova, H., Hofierka, J., Zlocha, M., \& Iverson, L.R. (1996). Modelling topographic potential for erosion and deposition using GIS. International journal of geographical information systems, 10(5), 629641.

33. Modeste, M., et al. (2016). "Cartographie Des Risques De L'erosion Hydrique Par L'equation Universelle Revisee Des Pertes En Sols, La Teledetection Et Les Sig Dans Le Bassin Versant De L'ourika (Haut Atlas, Maroc)." Eur Sci J 12.

34. Mrabet, R., et al. (2020). "La fertilisation améliore la production des prairies humides naturelles dans la montagne du haut Atlas oriental du Maroc: cas de la région d'Imilchil." African and Mediterranean Agricultural Research Journal-Al- Awamia(128).

35. Ouallali, A., Moukhchane, M., Aassoumi, H., Berrad, F., Dakir, I. (2016). The mapping of the soils' degradation state by adaptation the PAP/RAC guidelines in the watershed of Wadi Arbaa Ayacha, Western Rif, Morocco. J Geosci Environ Prot 4(07):77-82.

36. Rango, A. \& Arnoldus, H.M.J. (1987). Aménagement des bassins versants. In: Cahiers techniques de la FAO: 1-11.

37. Renard, KG., Foster, GR., Weesies, GA., \& Porter, JP. (1991) RUSLE: revised universal soil loss equation. J Soil Water Conserv 46(1):30-33 
38. Roose, E. (1996). Land husbandry - Components and strategy by Director of Soils Research. InFao Soil Bulletin. 70, p. 380.

39. Shin, G. (1999). "The analysis of soil erosion analysis in watershed using GIS." Department of Civil Engineering, Gang-won National University, Gangwon-do, South Korea, Ph. D. dissertation.

40. Wischmeier, W. H. \& Smith, D.D. (1978). Predicting rainfall erosion losses: a guide to conservation planning, Department of Agriculture, Science and Education Administration.

41. Yjjou, M., et al. (2014). "Modélisation de l'érosion hydrique via les SIG et l'équation universelle des pertes en sol au niveau du bassin versant de l'Oum Er-Rbia." The International Journal Of Engineering And Science (IJES) 3(8): 83-91.

42. Zingg, A. W. (1940). "Degree and length of land slope as it affects soil loss in run-off." Agric. Engng. 21: 59-64.

43. Zouagui, A., et al. (2018). "Modelisation du risque d'érosion hydrique par l'équation universelle des pertes en terre dans le Rif occidental: cas du bassin versant de Moulay Bouchta (Maroc)." European Scientific Journal 14(3): 1857-1788. 